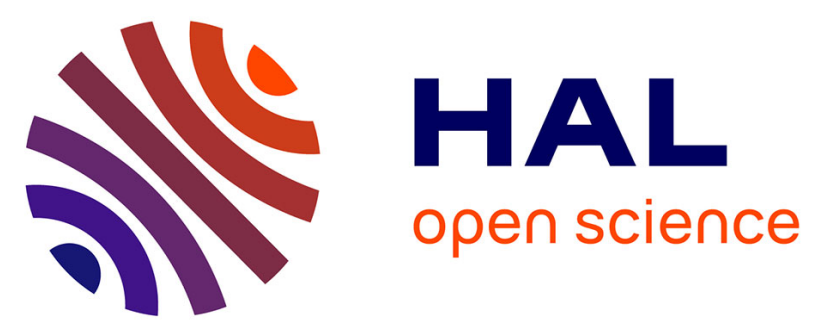

\title{
LyText : un environnement logiciel d'aide à la préparation de l'EAF (Epreuve anticipée de français du baccalauréat)
}

Jean-Marie Pierrel, Etienne Petitjean, Claire Etienne-Becker, Danielle Zitella, Véronique Zaercher-Keck

\section{To cite this version:}

Jean-Marie Pierrel, Etienne Petitjean, Claire Etienne-Becker, Danielle Zitella, Véronique ZaercherKeck. LyText: un environnement logiciel d'aide à la préparation de l'EAF (Epreuve anticipée de français du baccalauréat). Études de linguistique appliquée: revue de didactologie des languescultures, 2010, pp.15-30. hal-00523517

\section{HAL Id: hal-00523517 https://hal.science/hal-00523517}

Submitted on 5 Oct 2010

HAL is a multi-disciplinary open access archive for the deposit and dissemination of scientific research documents, whether they are published or not. The documents may come from teaching and research institutions in France or abroad, or from public or private research centers.
L'archive ouverte pluridisciplinaire HAL, est destinée au dépôt et à la diffusion de documents scientifiques de niveau recherche, publiés ou non, émanant des établissements d'enseignement et de recherche français ou étrangers, des laboratoires publics ou privés. 


\title{
LyText : un environnement logiciel d'aide à la préparation de l'EAF (Epreuve anticipée de français du baccalauréat) ${ }^{1}$
}

\begin{abstract}
Résumé
Dans cet article nous présentons le projet LyText (Lycée + Texte), un environnement informatique d'aide à la préparation du baccalauréat de français, développé par l'ATILF et en concertation étroite avec les enseignants grâce au soutien de l'Académie et de la Région Lorraine. Après une présentation générale de LyText, ses objectifs, sa philosophie générale, les connaissances mises en œuvre et un aperçu de la méthodologie suivie pour instancier un texte au sein de LyText, nous ferons un premier bilan de ses apports tant à l'enseignant pour l'accompagner dans ses objectifs pédagogiques qu'au lycéen comme support de travail personnel. Nous terminerons enfin en présentant les perspectives futures d'évolution de ce système.
\end{abstract}

\section{INTRODUCTION : LA GENESE DU PROJET}

Au cours des années 1999-2002, la région Lorraine développa le projet e-Lorraine qui permit la mise en place de salles informatiques équipées d'ordinateurs avec une connexion haut débit dans l'ensemble des lycées lorrains. Mais très vite il est apparu indispensable de mettre à disposition des élèves et des enseignants des ressources pédagogiques adaptées à leurs besoins pour répondre aux directives des programmes officiels qui, dès 2002, préconisent, dans le cadre de l'analyse de textes littéraires, l'emploi de ressources informatisées présentes au sein des CDI (Ministère 2002). C'est dans ce contexte que, depuis 2003, l'ATILF propose sur la plate-forme de l'Académie Nancy-Metz un ensemble de ressources développées au sein du laboratoire avec en particulier le Trésor de la Langue Française informatisé (TLFI: www.atilf.fr/tlfi) et la base textuelle FRANTEXT (www.atilf.fr/frantext). Cependant ces ressources (Pierrel 2005), quelles que soient leurs qualités et leurs richesses, ne sont pas véritablement adaptées aux besoins des lycéens. Ainsi est né le projet LyText (pour Lycée + Texte), un environnement informatique d'aide à la préparation du baccalauréat de français. Le développement de ce projet s'est mené en concertation étroite avec des enseignants de l'Académie et a conduit à la conception d'un outil logiciel utile aux enseignants et élèves pour la préparation du baccalauréat de français. Son l'usage aujourd'hui, après une phase de test et de validation en Lorraine, est en voie de généralisation au niveau national.

\section{PRESENTATION GENERALE DE LYTEXT}

\subsection{Objectif et approche générale de LYTEXT}

L'objectif de LyText étant d'apporter une ressource aux enseignants et une aide aux élèves en vue de la préparation de l'EAF (Epreuve Anticipée de Français du baccalauréat), plus particulièrement de l'épreuve orale, il convient de rappeler que le principal objectif de cette épreuve est d'évaluer la capacité de l'élève à mobiliser ses connaissances en vue de construire et d'argumenter sur sa compréhension et son interprétation d'un extrait de texte. L'examen consiste dans un premier temps à répondre à une question particulière portant sur un texte étudié en classe au cours de l'année scolaire. L'élève peut ainsi rendre compte de sa lecture en observant le texte et en menant une analyse simple de ses aspects essentiels. Dans un second temps, il s'agit pour l'élève d'avoir un entretien avec l'examinateur et de répondre à des questions sur l'œuvre intégrale d'où est extrait le texte ou sur le groupement de textes auquel ce texte appartenait.

Lors de la phase de conception de l'outil, les enseignants partenaires nous ont fait part de leur méthodologie d'approche et d'analyse des textes littéraires. Cette méthodologie, couplée avec les résultats de recherche en linguistique, stylistique ou encore compréhension de texte a permis de mettre au point le modèle qui propose différentes informations nécessaires à l’analyse du texte littéraire.

1 Projet réalisé en partenariat entre l'ATILF UMR Nancy-Université \& CNRS et l'Académie Nancy Metz avec le soutien de Nancy Université, du CNRS, du Conseil régional de Lorraine et des Ministères de l'Éducation nationale et de l'Enseignement supérieur et de la Recherche. 
Conscients que les méthodes d'approche du texte littéraire divergent selon les enseignants, nous avons conçu un modèle totalement paramétrable par l'usager qui lui permet de faire apparaître les grands types d'informations analytiques (structure, rythme, sonorités, figures de style, lexique, énonciation) dans l'ordre qu'il souhaite en fonction de son objectif. Il peut aussi neutraliser, pour les besoins d'une séquence d'enseignement par exemple, certaines informations qui n'apparaîtront pas alors sur les écrans des élèves. Ainsi, s'il souhaite faire travailler sa classe sur le repérage de la métaphore dans un texte particulier, il peut choisir de ne pas les faire afficher par LyText. Pour ce faire l'utilisateur de LyText dispose d'un panneau de contrôle lui permettant de présenter à l'écran un ensemble d'indices possibles. Ces divers indices, pouvant être superposés dans un écran de visualisation unique, restent alors distinguables grâce à divers codes couleur.

LyText se distingue nettement des annales de préparation au baccalauréat. En aucun cas nous ne proposons ici une interprétation du texte: nous avons choisi de nous limiter à la présentation interactive d'informations objectives contenues dans le texte, la construction de l'interprétation du texte restant à la charge de l'élève, guidé par son enseignant lors d'une utilisation en classe, ou en autonomie lors d'un usage personnel en dehors de la classe (Becker, Faure 2008).

La philosophie générale de constitution de la base de textes sur laquelle s'appuie LyText est claire: forts de notre conviction qu'il revient aux enseignants pédagogues de déterminer les informations qu'ils jugent pertinentes pour l'étude d'un texte et compte-tenu de leurs objectifs pédagogiques, nous avons mis en place, en accord avec l'Académie, une politique de mutualisation à l'image de ce que nous avons déjà mis en œuvre dans d'autres projets (Pierrel 2008). Après une phase initiale menée en concertation entre le laboratoire et les enseignants, au cours de laquelle les connaissances mises en œuvre dans LyText ainsi que la structure générale du système ont été spécifiées (cf. § 2.2), les enseignants travaillant avec LyText assurent la préparation des textes qui sont ensuite codés informatiquement par le laboratoire (cf. § 3) et intégrés au système avant l'utilisation effective par les enseignants et les élèves (cf. § 4).

Actuellement, au terme de la première phase d'expérimentation, LyText regroupe 43 textes correspondant à des grands classiques que peuvent être amenés à utiliser les enseignants, en conformité avec les objets d'étude fixés par les instructions officielles dans le cadre de la préparation de l'EAF. L'un de nos objectifs à court terme est de pouvoir proposer une base d'au moins 100 textes.

\subsection{Les connaissances mises en œuvre dans LyText}

Dans un premier temps, à travers un écran de contrôle l'élève peut choisir un texte à étudier en le sélectionnant à travers divers critères : nom de l'auteur, mots du titre, objets d'étude, thèmes, registre ou dates. Supposons qu'au terme de cette première étape l'élève ait sélectionné Bohémiens en voyage de Charles Baudelaire, un écran dont on trouvera un exemple en figure 1 apparaît alors.

Dès ce premier écran l'élève dispose d'un module de contrôle lui permettant de faire apparaître sous l'onglet « module » :

- des questions à traiter,

- des axes de lecture possibles,

- des informations génériques regroupées sous la rubrique «instructions officielles » qui permettent à l'élève de replacer le texte dans un contexte plus général et de situer l'extrait en termes d'objets d'étude (ici la poésie), de thèmes (poète, réalité, voyage dans ce cas) ou de registres (lyrique pour les « Bohémiens en voyage »).

- des informations complémentaires, sous l'onglet «interculturel» (en l'occurrence dans ce cas la reproduction de la gravure "Les bohémiens en marche : l'arrière garde » extrait de "Les bohémiens » de Jacques Callot (1592-1635) », », accompagnée d'un texte indiquant que ce poème a été inspiré de cette gravure

Si l'on fait abstraction de cette composante «modules », la partie la plus importante concerne la structuration des informations analytiques (Pelissier \& Becker 2005) qui sont mises à disposition des usagers de LyText. Ces informations analytiques sont accessibles à travers l'onglet «texte » et sont associées aux informations caractérisant des mots ou des groupes de mots dans le texte. Elles sont regroupées suivant six grands types d'informations. : 
- la structure : ses différentes parties (thèse, arguments, exemple...), sa forme (sonnet, ode...), ses connecteurs (temporels, spatiaux, argumentatifs...),

- les effets de rythme : sa prosodie (coupe, césure...), ses constructions syntaxiques particulières (binaire, ternaire,...), sa métrique (nombre de pieds...),

- les sonorités : les rimes, les paronomases, les allitérations, les assonances...,

- certaines figures de style : les métaphores, les métonymies, les personnifications...,

- le lexique : la définition de mots difficiles extraite du TLFi (en l’occurrence, ici, la définition pertinente du mot en fonction du contexte), les champs lexicaux...,

- l'énonciation : les types de phrase, les pronoms personnels, les modalisateurs...

Ces informations peuvent être présentées à la demande et en superposition sur le texte comme aide aux élèves pour construire et argumenter leur interprétation du texte.

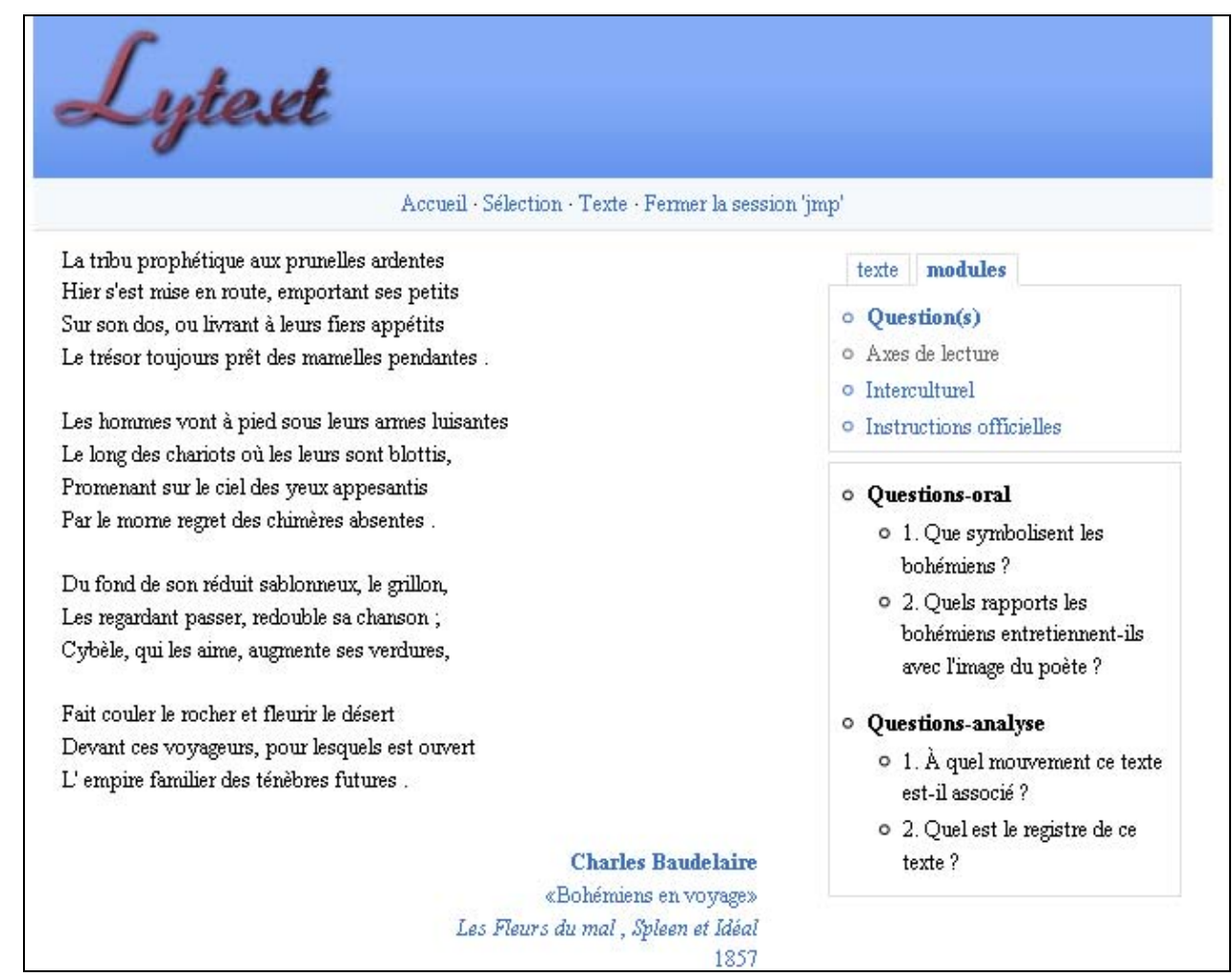

Figure 1 : écran général de LyText

A titre d'exemple, la figure 2 présente une superposition d'informations sur le lexique : champs lexicaux majeurs du texte (ici voyage en rouge, nature en mauve et tristesse en bleu) et définitions de mots considérés par l'enseignant comme problématiques et repérables par la marque $\mathbf{0}$. Une simple sélection sur cette marque permet alors de présenter la définition du mot en contexte issue du TLFi.

Quant à la figure 3 elle présente un écran superposant des informations de structure et de rythme : structure du sonnet en quatrains et tercets, rimes, prosodie à travers des marques verticales en bleu et des informations sur les figures de style présentes dans le texte, telles, ici, les enjambements marqués en italique, une métaphore surlignée en mauve ou des oxymores surlignés en vert.

\section{L'INSTANCIATION D'UN TEXTE AU SEIN DE LYTEXT}

\subsection{Préparation du texte par l'enseignant}

Le travail préparatoire d'analyse des textes en vue de leur insertion dans la plateforme LyText est effectué par les enseignants. Chaque texte est décrit dans un document Word qui sert ensuite de base à l'informatisation des données. 
Accueil - Sélection · Texte · Fermer la session 'jmp'

La tribu prophétique (3) aux prunelles (7) ardentes

Hier s'est mise en route, emportant ses petits

Sur son dos, ou livrant à leurs fiers (7) appétits

Le trésor toujours prêt des mamelles pendantes.

Les hommes vont à pied sous leurs armes luisantes

Le long des chariots où les leurs sont blottis,

Promenant sur le ciel des yeux appesantis

Par le mome regret des chimères (3) absentes.

Du fond de son réduit (3) sablonnueus Définition

Les regardant passer, redouble sa cha Chimère : 1 . Rêverie quelque peu

Cybèle 9 , qui les aime, augmente se folle. 2. Illusion. (TLFi)

Fait couler le rocher et fleurir le désert

Devant ces voyageurs, pour lesquels est ouvert

L' empire familier des ténèbres futures.

texte modules
- Structure
- Rythrue
- Sonorités
- Figures de style
- Lexique
- Sens des mots
- Champs lexicaux
$\quad$ voyage
o nature
○ tristesse
Cornotation
- Enonciation

Figure 2 : informations lexicales

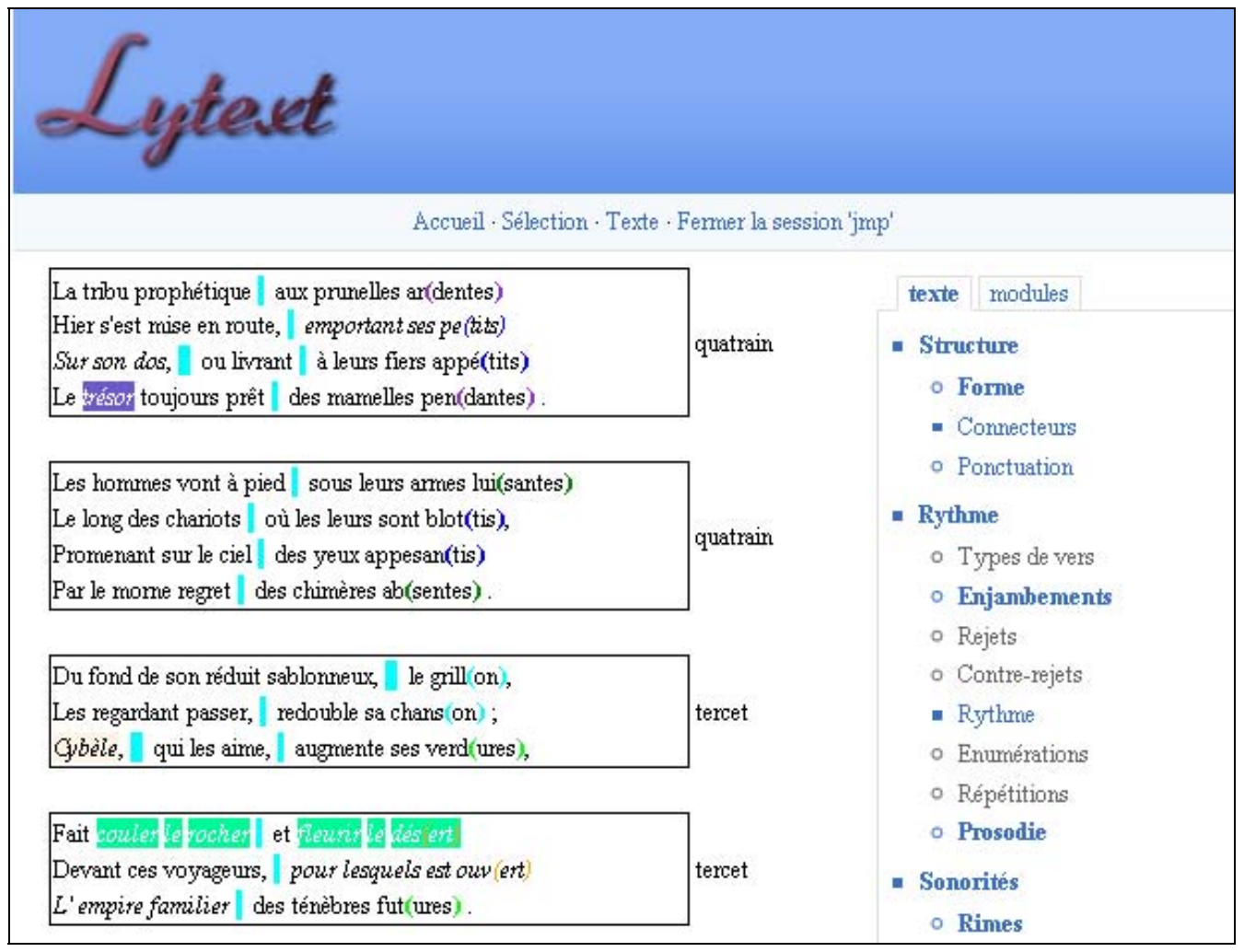

Figure 3 : informations de structure et de rythme

Les premières informations apportées par les enseignants concernent les métadonnées (données à propos du texte). Celles-ci donnent des renseignements sur le nom de l'auteur, de l'œuvre, la date de parution ou encore les thèmes abordés dans l'extrait ainsi que les registres et les objets d'étude.

La suite du travail de l'enseignant consiste à faire émerger graphiquement dans le document de travail Word toutes les informations importantes résultant de son analyse du texte. Pour chaque type d'informations (structure du texte, champs lexicaux, figures de style, mots-clés, etc.), une copie du texte analysé est insérée dans le document de travail et l'enseignant surligne de différentes couleurs 
toutes les informations pertinentes qui devront être codées au niveau informatique par la suite. L'analyse des champs lexicaux pourra se traduire ainsi :

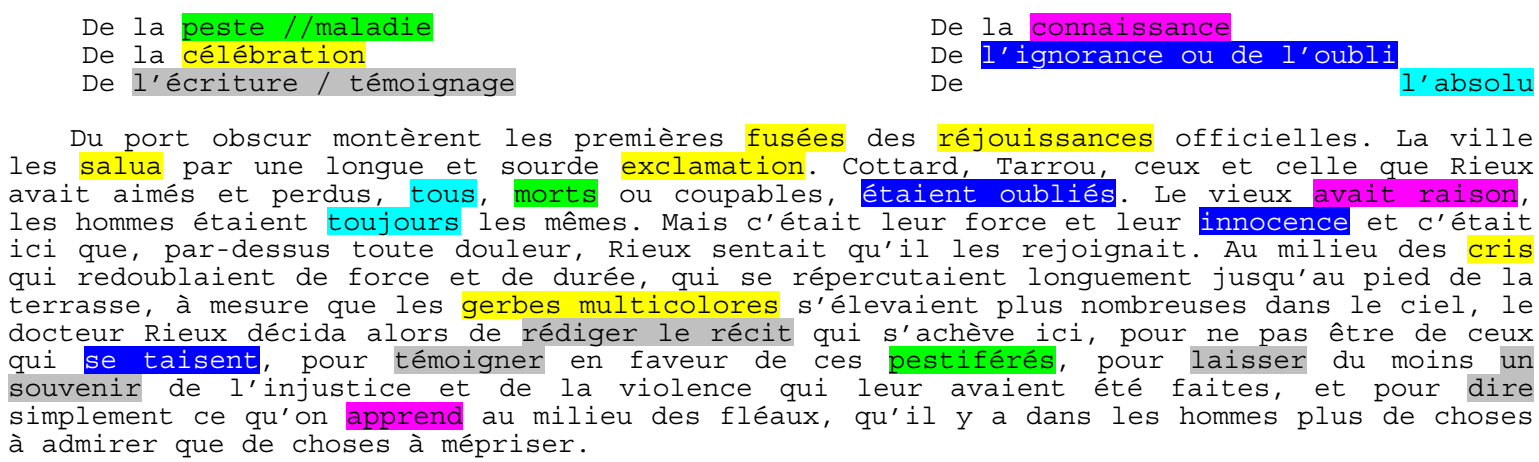

Une fois toutes ces informations surlignées dans le document, les derniers renseignements à fournir concernent les questions d'analyse et d'oral associées au texte. Le document final est ensuite transmis au laboratoire pour être informatisé et intégré dans la plateforme LyText.

\subsection{Codage des informations et intégration dans le logiciel}

Cette étape consiste à coder les informations données par les enseignants dans un langage exploitable par un programme informatique. Pour les besoins de ce projet, nous avons utilisé un langage de balisage de données. Le langage XML (eXtended Markup Language) est une recommandation du W3C (World Wide Web Consortium) proposant un modèle générique de structuration de données avec un système de balises. Chaque balise utilisée dans un document XML possède une sémantique qui lui est propre. La structure d'un document est définie en fonction des besoins spécifiques du projet. Pour LyText, nous avons utilisé un schéma appelé DTD (Document Type Definition) adapté pour permettre le codage de toutes les informations nécessaires au bon fonctionnement du logiciel.

Les balises XML permettent d'isoler et de repérer les données en leur associant un nom. De même que pour les métadonnées, les informations d'analyse du texte sont également codées avec des balises XML spécifiques comme dans l'exemple de phrase ci-dessous :

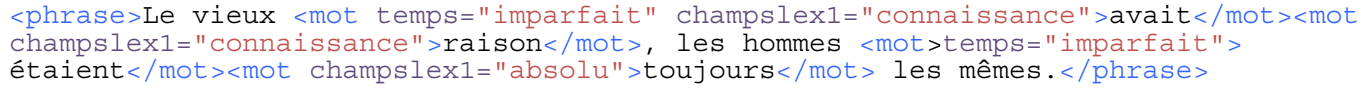

Cet exemple permet de constater que le texte est découpé en phrases puis en mots. Chaque mot est codé avec une balise <mot> pouvant contenir une ou plusieurs valeurs résultant de l'analyse de l'enseignant. Ainsi le mot « avait » est codé avec deux informations : le temps du verbe (imparfait) et le champ lexical (connaissance) dont fait partie le mot « avait » à cet endroit dans le texte. Le codage informatique des textes peut devenir relativement complexe car on constate qu'un mot dans le texte peut contenir jusqu'à une dizaine d'informations tirées des analyses du texte. Cette opération de codage nécessite par conséquent une validation a posteriori pour vérifier la cohérence des opérations saisies. Une fois le document saisi au format XML et vérifié, la dernière opération consiste à l'introduire dans la plateforme LyText. Un simple clic suffit à ajouter automatiquement le nouveau texte. Il est alors visible et disponible immédiatement dans LyText.

\section{UTILISATION DE LYTEXT}

\subsection{Utilisation en classe}

Recourir à LyText permet à l'enseignant de proposer à ses élèves de construire une explication de texte de manière à renouveler l'approche de cet exercice. Plusieurs façons de procéder sont possibles :

Le professeur seul manipule LyText en situation de vidéo-projection et conduit sa classe à découvrir le texte qu'il a choisi et à lui donner sens. Deux situations sont envisageables : (i) les élèves disposent sur leur table de travail à la fois d'un polycopié de l'extrait du texte vidéo-projeté qu'ils renseignent au fur et à mesure de l'explication et d'une feuille de cours qui va leur permettre de 
conserver une trace plus détaillée de la séance menée. (ii) Les élèves sont en salle informatique, ils ont sur leur poste de travail le texte vidéo-projeté et le complètent au fil de l'explication en recourant aux fonctionnalités du traitement de texte.

Le professeur et ses élèves manipulent LyText. Deux manières de mener le cours s'offrent à l'enseignant : (i) les élèves sont en salle informatique et accèdent grâce à LyText à différentes informations qui vont leur permettre à la fois de répondre aux questions posées par leur professeur qui dirige la séance d'explication et de proposer leur interprétation du texte. Au fur et à mesure de l'avancée dans le sens à donner à l'extrait étudié, chaque élève complétera sa feuille de cours et le polycopié de l'extrait de texte étudié. (ii) Les élèves, toujours en salle informatique et en situation avec LyText, renseignent en même temps sur traitement de texte l'extrait expliqué en suivant les directives de l'enseignant.

LyText permet ainsi d'aborder l'explication de texte de manière différente, aussi bien pour l'élève que pour l'enseignant.

Les élèves se positionnent volontiers en acteurs du sens et interviennent plus aisément en raison de l'utilisation d'un outil de travail qui leur correspond. L'échange au sein de la classe gagne du coup en intérêt et en dynamisme. L'environnement proposé par LyText motive donc l'élève mais le met également en confiance, ce qui est essentiel : il se dit qu'il peut parvenir à proposer une analyse du texte en utilisant les éléments déjà à sa disposition. L'outil est par conséquent de nature à inciter plus volontiers à la recherche, à la réflexion. Il donne envie à l'élève de trouver des solutions. C'est comme si ce dernier se retrouvait en situation de mener une enquête. La méthode d'exploration du sens peut être différente selon chacun et LyText offre cette possibilité : soit l'élève s'appuie sur les relevés pour en déduire les enjeux du texte, soit il émet des hypothèses de lecture qui vont être corroborées par ces mêmes relevés. Dans les deux cas, la recherche devient passionnante. Chaque élève y trouve dès lors son compte selon la manière dont il préfère procéder. En outre, les élèves sont sensibles à la présence de données propres à les éclairer sur le sens à donner à un texte. Prenons pour exemple l'énonciation : les relevés proposés invitent à s'interroger sur la présence du narrateur ou de l'auteur dans une oeuvre. Souvent, les élèves négligent cette piste car ils en ignorent l'intérêt ou ne savent pas ce que l'on met derrière ce mot. L'objectif est ici double: comprendre ce que l'on doit chercher quand on parle d'énonciation et réfléchir à la fonction de cette donnée dans l'extrait. Ainsi l'élève s'habitue grâce à LyText à une démarche d'analyse qu'il va pouvoir facilement exploiter par ailleurs, notamment lors des épreuves anticipées de français.

L'enseignant quant à lui prépare son explication de texte en s'appuyant sur les données déjà existantes dans LyText. Celles-ci vont lui permettre d'orienter l'étude de l'extrait selon ce qu'il aura choisi de travailler plus particulièrement lors de la séance. Ainsi, s'il désire attirer l'attention des élèves sur les procédés mis en œuvre dans un texte pour convaincre ou persuader, il travaillera plus particulièrement autour de la structure, du rythme, des figures de style et de l'énonciation. Il sera du coup intéressant de démontrer aux élèves que le simple relevé de figures de style par exemple ne permet pas de donner sens au texte s'il n'est pas accompagné d'une analyse, d'un commentaire. Le fait que ces relevés existent déjà oblige l'élève à aller plus loin alors qu'avant il pouvait considérer avoir effectué son travail en indiquant simplement des comparaisons, des anaphores ou même des métaphores. LyText permet à l'enseignant d'inciter l'élève au commentaire de texte, le préparant ainsi aux épreuves écrites du baccalauréat. En outre, on peut prolonger l'exercice d'explication en demandant aux élèves de répondre aux questions apparaissant en partie Modules. C'est l'occasion de faire un bilan de lecture et de vérifier la bonne compréhension du texte, voire de prolonger la réflexion. A l'issue d'une explication, l'enseignant peut envisager de se servir de cette partie Modules dans le but de préparer l'élève à l'épreuve orale du baccalauréat de français, ce qui sera l'occasion d'apporter des éclaircissements sur les exigences de cet exercice oral.

\subsection{Utilisation autonome par l'élève}

L'élève peut travailler à partir de LyText de manière autonome dans différentes situations.

En classe, LyText peut être utilisé de plusieurs manières en fonction des objectifs du professeur : (i) l'enseignant en salle informatique propose aux élèves un temps de travail, par groupe limité à une 
durée de quinze à vingt minutes, au cours duquel il leur demande de noter sur feuille leurs premières impressions de lecture d'un texte qu'il va ensuite étudier. Les élèves recourent à LyText et peuvent déjà commencer à explorer l'extrait qui sera analysé. L'échange qui suivra entre la classe et le professeur sera plus riche, plus dense et permettra sans doute d'atteindre les objectifs plus rapidement grâce à ce temps de réflexion qui aura été donné à partir de LyText. (ii) A l'issue d'une explication de texte, l'élève produit sa propre conclusion en s'aidant de la partie Modules. Ce temps de recherche personnelle est essentiel pour s'approprier tout ce qui aura été vu au cours de la séance. L'élève peut ainsi se rassurer sur son approche du texte et l'enseignant peut vérifier la bonne compréhension par la classe de l'explication menée. (iii) Lors d'un devoir bilan situé en fin de séquence, l'enseignant peut choisir de permettre aux élèves d'accéder au groupement de textes vus dans LyText. Il peut s'agir d'une question de synthèse pour laquelle recourir aux textes sera utile. LyText et son environnement les y aidera et leur permettra également de comprendre la nécessité de maîtriser parfaitement ses explications, de connaître même par cœur certains passages des textes pour alimenter, développer et affiner sa réflexion. En outre, cette manière de procéder peut dans un premier temps aider les élèves les plus faibles en les mettant en confiance.

A la maison, il est possible de proposer à l'élève de recourir à LyText dans les cas suivants: (i) Pour lui permettre de réviser de manière plus attrayante les lectures analytiques menées dans le cadre de la préparation aux EAF. Se référer aux questions type pouvant être posées à l'oral peut constituer un bon exercice d'entraînement à la maison. (ii) Pour retrouver plus facilement le fil conducteur d'une explication, ce qui constitue une aide précieuse pour un élève qui aurait été absent lors d'une séance. (iii) Comme support appréciable pour l'élève dans le cas où l'enseignant aurait choisi de proposer un devoir de commentaire littéraire d'un texte figurant dans LyText. (iv) A l'issue de l'étude d'une œuvre intégrale, l'enseignant peut puiser dans LyText pour demander aux élèves à la fois de procéder à des lectures complémentaires et de réaliser un travail de synthèse à partir des données qu'ils trouveront sur les textes.

Au final, LyText aide l'élève à gagner en autonomie dans sa propre réflexion face au texte car il intègre un mode opératoire et des mécanismes de recherche qui vont lui être utiles pour aborder plus efficacement un extrait à étudier.

\subsection{Premier bilan de l'usage effectif de LyText}

Par définition, toute ressource pédagogique est mise à la disposition des professeurs et c'est à eux qu'il revient de décider dans quelle mesure ils souhaitent l'utiliser et peuvent l'adapter aux objectifs pédagogiques fixés à une séance d'analyse de texte. Il n'existe pas actuellement d'autre ressource de même nature que LyText à la fois pour l'enseignement du français au lycée et pour l'entraînement des élèves à l'EAF. La base LyText, en ce qu'elle est innovante, conduit donc le professeur utilisateur à un travail de préparation spécifique, en amont de la séance : il porte sur l'articulation entre les pistes d'analyse littéraire à exploiter précisément pour un texte et sur la plus-value pédagogique qui peut être obtenue à partir du mode d'accès aux informations contenues dans LyText. Cette question de la plusvalue est essentielle en ce qu'elle constitue l'argument principal qui favorise ou, au contraire, peut freiner l'utilisation d'une ressource numérique.

Dans l'Académie de Nancy-Metz, la mise à disposition de LyText a été accompagnée de deux façons. Il a paru tout d'abord indispensable d'en faciliter l'accès grâce à l'environnement numérique de travail PLACE (Plateforme Lorraine d'Accessibilité et de Communication pour l'Education) qui est implanté dans l'ensemble des lycées généraux et technologiques lorrains et dans lequel chaque professeur dispose de LyText. Ainsi la connexion est-elle rendue aisée depuis toute salle équipée d'un ordinateur dans l'établissement scolaire et notamment pour une utilisation en séance de cours. En outre, la préparation de la séance est elle-même rendue très pratique du fait que le professeur a la possibilité de se connecter depuis son domicile à LyText et que l'ENT lui offre la possibilité de stocker ses documents.

Se contenter de mettre LyText à la disposition des professeurs ne pouvait seul suffire et les membres des corps d'inspection (CTICE et IA-IPR de lettres) impliqués dans ce projet ont jugé indispensable d'organiser des présentations pour montrer de façon concrète comment l'utilisation des différentes catégories d'informations contenues dans LyText se veut souple et variée, ce qui a 
notamment pris la forme de démonstrations réalisées par un professeur et commentées écran par écran. Il ne s'agissait nullement de présenter une séance toute prête mais bien de proposer des pistes pédagogiques : usages possibles dans le cadre de la lecture analytique en classe de première mais aussi en classe de seconde, certains textes contenus dans la base pouvant effectivement être adaptés aux objets d'étude de ce niveau d'enseignement; réinvestissements et liens possibles entre la méthodologie de la lecture analytique et celle du commentaire littéraire ; pratiques envisageables en classe entière avec projection des informations grâce à un tableau blanc interactif ou utilisation autonome par l'élève en vue d'un entraînement individuel à l'épreuve orale de l'EAF. Ainsi LyText at-il été d'emblée associé à un contenu pédagogique, modulable selon les démarches et les objectifs choisis par le professeur, et certaines des réticences exprimées à propos de l'aspect très technique des informations projetées à l'écran ont été, dans une large mesure, plus aisément surmontées.

Une évaluation des usages de LyText est prévue au cours de l'année scolaire 2010-2011 et devra s'appuyer sur une double orientation. D'une part, il sera intéressant d'observer à quelle fréquence les professeurs utilisent une ressource qui, rappelons-le, dans le principe, ne peut qu'être proposée. L’intérêt alors, outre celui qui est lié à la spécificité de LyText, sera de disposer d'informations plus larges sur l'intégration des outils informatiques dans la séance de cours de français. D'autre part, les démarches pensées par les professeurs gagneront à être recueillies dans la mesure où elles permettront de connaître des usages au sein de la classe et de mesurer les possibles bénéfices qui peuvent être obtenus de ce mode d'entraînement des élèves à l’EAF.

\section{PERSPECTIVES}

Les politiques publiques contribuent à généraliser l'usage des technologies de l'information et de la communication dans l'enseignement. Les établissements d'enseignement scolaire et supérieur ont enregistré une amélioration sensible de leur taux d'équipement en micro-ordinateurs et de leur accès Internet, y compris avec des connexions à haut débit, posant avec plus d'acuité encore la question des ressources à mettre à la disposition des élèves, des étudiants et des enseignants. Pour y répondre, le Ministère de l'Education Nationale et le Ministère de l'Enseignement supérieur et de la Recherche mènent une politique active de soutien au développement des ressources multimédias éducatives afin de permettre à la communauté éducative de disposer de produits de qualité correspondant à ses attentes et aux orientations du système éducatif. Dans ce cadre, au terme de la première phase d'expérimentation et forts de l'intérêt manifesté tant par les enseignants que par les élèves ou l'Académie, les Ministères ont décidé de soutenir l'ATILF pour, en particulier, participer au développement de LyText. Les Ministères apportent leur soutien par une dotation au titre des actions spécifiques, en vue de l'enrichissement de la base de textes de LyText et de l'évolution de son interface afin d'en faciliter l'usage sur les nouveaux outils pédagogiques (tableaux numériques interactifs et tablettes électroniques).

Nos objectifs dans ce cadre au cours de l'année à venir sont les suivants :

- enrichir la base de textes de LyText en concertation avec l'Académie Nancy-Metz et ses enseignants pour parvenir à une centaine de textes disponibles, correspondant à des textes classiques proposés par les enseignants dans le cadre de la préparation de l’EAF,

- apporter des améliorations à l'interface en concertation et en interaction avec des experts pédagogiques de l'Académie Nancy-Metz, en prenant en compte les expérimentations faites à ce jour,

- réfléchir à une adaptation de LyText pour des supports de type e-book dont on peut penser que l’usage se généralisera dans les années à venir,

- mettre à disposition gratuitement et de manière permanente un corpus de textes numérisés à destination des enseignants, des élèves et des étudiants, par le biais de la plateforme de l'Académie Nancy-Metz avec possibilité de téléchargement, après enregistrement de l'élève ou de l'enseignant, pour des usages par exemple sur e-book) ${ }^{2}$.

2 Une telle limitation est nécessaire afin, dans le cadre de l'exception prévue par la loi pour les besoins d'enseignement, de respecter les droits d'auteurs et d'éditeurs des textes proposés au sein de LyText. 
- contribuer en concertation avec les services des académies à la généralisation progressive de l'usage de LyText, par des actions de communication concertées.

Par ailleurs sur le plan de la recherche, nous souhaitons poursuivre nos travaux afin de proposer à terme aux enseignants une version leur permettant d'alléger leurs tâches de préparation de textes à travers l'usage de composants logiciels de traitement automatique des langues leur proposant des balisages automatiques d'une partie importante des informations utilisées au sein de LyText. Outre le choix de la bonne définition d'un mot, pertinente en fonction du contexte, extraite des données lexicales de l'ATILF, et en particulier du TLFi (la thèse de Claire Etienne-Becker propose en effet un algorithme informatique pour un tel choix), nos efforts porteront dans un premier temps sur le balisage des connecteurs, des pronoms personnels, des démonstratifs et des déictiques, des modalisateurs, de la métrique, des rimes, etc., autant de domaines pour lesquels, au vu des résultats actuels, nous pensons pouvoir rapidement exhiber des algorithmes performants.

Sur ces points il convient de noter qu'en aucun cas nous n'envisageons de proposer un système entièrement automatique. Le lecteur l'aura compris, l'orientation que nous poursuivons dans LyText est de placer l'outil informatique au service de la pédagogie et de l'enseignant et en aucun cas comme alternative. L'ensemble des propositions que pourra faire un tel système d'aide au balisage automatique d'informations dans un texte littéraire, tout comme l'ensemble des préparations, devront être validées préalablement par les enseignants et l'inspection pédagogique avant leur intégration finale dans le système. C'est, nous semble-t-il, indispensable pour maintenir à l'avenir la prééminence du pédagogique sur l'outil et ainsi continuer à défendre un enseignement du français de qualité tout en intégrant au mieux l'usage des techniques actuelles de l'information et de la communication.

\section{BIBLIOGRAPHIE}

BECKER C. FAURE P. «LyText : une approche de l'analyse des textes littéraires sur mesure », Les Dossiers de l’Ingénierie Pédagogique, SCEREN-CNDP, 2008.

MINISTERE 2002a. Programmes Français, classe de seconde. Collection Lycée voie générale et technologique, CRDP, Paris

PIERREL, J.M. 2005. «Un ensemble de ressources de référence pour l'étude du français: TLFi, FRANTEXT et le logiciel STELLA », Revue Québécoise de Linguistique, volume 32/1, , p. 155176, 2005

PIERREL J.M. 2008. «De la nécessité et de l'intérêt d’une mutualisation informatique de connaissances sur le lexique de notre langue », 1er Congrès Mondial de Linguistique Française, Paris juillet 2008, article disponible sous: http://www.linguistiquefrancaise.org et http://dx.doi.org/10.1051/cmlf08330

PELISSIER C. BECKER C. 2005. «Le modèle de connaissance dans LyText : un environnement informatique d'aide à la préparation du baccalauréat », 1er colloque international de didactique DidCog 2005. 26-28 janvier 2005, France

Jean-Marie PIERREL, directeur de l'ATILF (Nancy Université \& CNRS)

Claire ETIENNE-BECKER, doctorante ATILF

Etienne PETITJEAN, ingénieur de recherche CNRS ATILF

Véronique ZAERCHER-KECK, IA-IPR de Lettres (Académie de Nancy-Metz)

Danielle ZITELLA, professeur de Lettres au lycée Jean-Hanzelet de Pont à Mousson 